
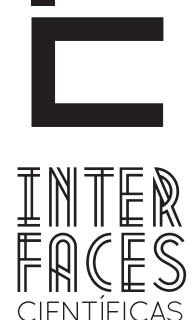

HUMANASE SOCIAIS

\title{
PARADIGMAS QUANTITATIVO E QUALITATIVO NO COTIDIANO DA INVESTIGAÇ̃̃̃O
}

\section{Diego Rodrigues \\ Lorena Monteiro ${ }^{3}$}

\section{RESUMO}

0 presente ensaio traz a discussão o emprego das metodologias qualitativa e quantitativa de análise para pesquisas na área das ciências sociais. Pontuamos alguns dos preconceitos clássicos de rejeição a um e outro paradigma, com o objetivo de desmistificar elementos de recusa e a frisar os pontos de colaboração das duas frentes metodológicas no exercício científico.

\section{PALAVRAS-CHAVE}

Metodologia. Método Quantitativo. Método Qualitativo.

\section{Marina Melo $^{2}$}

\section{ABSTRACT}

This essay brings the issue the use of qualitative and quantitative methods of analysis for social science researches. It concerns about some of the classic prejudices and rejection to different paradigms, demystifying refusal elements and emphasizes the collaboration points of the two methodological means in the scientific exercise.

\section{KEYWORDS}

Methodology. Quantitative Method. Qualitative Method. 


\section{RESUMEN}

Este ensayo lleva a la discusión del uso de métodos cualitativos y cuantitativos de análisis investigativas en las ciencias sociales. Señalamos algunos de los prejuicios clásicos a los dos paradigmas, desmitificando los elementos de rechazo y mirando los puntos de colaboración entre los dos paradigmas como un ejercicio metodológico científico.

\section{BREVÍSSIMA APRESENTAÇÃO DA IDEIA DE DE- BATE: POR QUE PENSAMOS NISSO?}

0 presente texto fundamenta-se em alguma experiência que os autores tiveram, enquanto docentes da Faculdade Integrada Tiradentes - FITs, na matéria "Metodologia Científica". Embora tal disciplina seja de ampla oferta em diversas áreas do saber, detemo-nos neste ensaio às questões que circunscrevem os pontos básicos de distinção entre metodologias

\section{PALAVRAS CLAVE}

Metodología. Método Cuantitativo. Método Cualitativo. qualitativa e quantitativa, a utilizar tais paradigmas como alicerces fundamentais na elaboração de projetos. Demonstramos, por suposto, a necessidade de domínio e compreensão das duas esferas de investigação, exemplificando possibilidades de metodologias mistas.

\section{MATEMÁTICA E CIÊNCIAS SOCIAIS: PROXIMI- DADE NAQUILO QUE PARECE DISTANTE}

Antes de adentrarmos nas possibilidades oferecidas pelos paradigmas qualitativo e quantitativo, faremos uma interconexão entre a matemática (e a estatística precisamente) e as ciências humanas, a demonstrar como embora as ciências humanas carreguem um estereótipo acadêmico de "anti-numérica", os universos de letras e números apresentam-se muito mais próximo, e simples, do que em geral se supõe.

A história da matemática nas ciências humanas antecede a história da sociologia, da ciência política ou das ciências sociais amplamente. A relação entre quantidade de informação e sabedoria, a definição de arquivos na lógica matemática, a matemática no desenvolvimento do raciocínio científico moderno ou a importância da escrita matemática na sensação de poder-se mobilizar a totalidade dos objetos são tópicos de discussões demasiadamente antigas (Ferreira, 2003). Desde a remota civilização egípcia já temos registros que se aproximam das relações que hoje tentamos imaginar entre ciências sociais e a matemática, quando governantes colhiam dados sobre seus súditos para preservação do poder. No século XVI, temos as primeiras análises sistemáticas de fenômenos 
sociais, como batizados, casamentos e funerais. 0 Iluminismo, ao trazer ao debate as noções de esclarecimento, debelação de tutela, razão e autonomia, traz também a importância do modelo matemático como elemento sintético de razão, especialmente ao dotar o pensamento descartiano e sua lógica cartesiana como fundamentais para a filosofia moderna. As ciências sociais não trazem consigo o emprego das técnicas matemáticas: não só a matemática lhe é essencialmente anterior, como a sua aplicação nas ciências humanas e na filosofia. Na relação das ciências sociais com o que entendemos hoje por estatística, uma parte da matemática aplicada, temos intelectuais como John Graund (século XVII) e Godofredo Achenwall (século XVIII) que buscaram sintetizar então modelos estatísticos numa disciplina, como estabelecer as relações desta com as demais ciências nos objetivos de descrição, explicação e exploração.

Thomas Wilson (1999) em "Sociologia e o Método Matemático", publicado em Teoria Social Hoje, argumenta que a matemática deve ter um papel heurístico no desenvolvimento das ciências sociais. Wilson demonstra como o modelo das ciências naturais não pode ser aplicado diretamente às ciências sociais haja vista as diferenças essenciais entre os dois universos de conhecimento. Mostra-nos como a matemática, desde Galileu, tem sido um idioma universal do mundo científico, sobretudo quando da contribuição de clássicos como Copérnico, Kepler e Newton. Condorcet, por exemplo, se ocupou de conhecer os fenômenos sociais através da matemática. A ideia de que as ciências sociais deveriam seguir os mesmo moldes das ciências naturais já foi ponto demasiadamente protagonizado nas ciências sociais, formulada como tese explícita nos pensamentos de Augusto Comte e Stuart Mill, quando já se falava da complexidade dos fenômenos sociais, em que as ciências sociais eram vistas e formuladas como as ciências naturais da sociedade. Tal postulado tornou-se uma postura metodológica ortodoxa em alguns contextos, sobretudo no campo da Economia. Wilson tece sobre a diversidade do uso da matemática e disserta sobre a doutrinal ex- tensional, idioma base das ciências naturais, na qual existiriam assertivas verdadeiras ou falsas, no que os cientistas deveriam se preocupar somente com as verdadeiras para investigações mais profundas. Seria o extensionalismo uma lógica sintética de objetividade nas ciências naturais, sustentado pela lógica-padrão, sua linguagem e ideia capitais. Contudo, e a contraponto, o paradigma da intensividade vem dizer que a maior dificuldade do extensionalismo é que este fica preso a conceitos que não podem ser extensionalmente formulados, o que o amarra a uma contradição interna. (A exemplo, como lidar com uma sentença de tipo “essa assertiva é falsa”?). Logo, a perspectiva da intensividade coloca que nem todo modelo das ciências naturais pode ser aplicado às ciências sociais. Wilson argumenta que não é, sobremaneira, contra o uso de métodos matemáticos nas ciências sociais, mas que entende que tais métodos não podem seguir a mesma aplicação das ciências naturais, no que a matemática deve cumprir um papel heurístico nas ciências sociais por nesta conter fenômenos particularmente distintos.

Chalmers (1994) demonstra como a estatística tomara cena no panorama inglês ao mesmo ritmo dos estudos de teorias eugenistas, de dominação de uma classe média profissional. Teóricos como Pearson estabeleceram critérios de correlação para variáveis numéricas importantes. Já Yule, criara testes de tabelas $2 \times 2$ para variáveis qualitativas a partir da preocupação com frequências. Interessante notar que os interesses de Yule não eram centrados na eugenia, mas sim, em estudos de populações pobres e demais fenômenos sociais. Entretanto, os estudos de Pearson não se limitaram à eugenia, tendo ele orientado trabalhos diversos com recursos estatísticos, a exemplo de Gosset, que utiliza seus métodos para compreender a produção fabril, como da cervejaria irlandesa Guiness. 0 que Chalmers chama à atenção é que estes processos de legitimação e tentativa de afirmação da estatística nas ciências sociais vêm com um conjunto de interesses das instituições de que estes teóricos faziam parte. "A estatística tinha interesses especí- 
ficos que a sustentava" (Chalmers, 1994). Por outro lado, em um cenário estadunidense, poderíamos pensar nas contribuições de Stouffer e Lazarsfeld. Stouffer dedicou-se a estudos sobre depressão nos EUA, ao status dos negros na década de 1930 e ao sucesso dos EUA na II Guerra Mundial. Contribuiu para estudos de organização de dados, questionários, análises etc. Paul Lazarsfeld, por sua vez, traz demais razões lógicas para os estudos de Stouffer sobre a Guerra e pesquisa fenômenos de comunicação, comportamento econômico e profissões. Além de criar os modelos de elaboração pelos quais demonstra e nomeia o comportamento de correlações bivariadas face à introdução de variáveis de controle, aprofunda estudos longitudinais de painel e cria Centros de Investigação na área das profissões, bem como o Centro Permanente de Pesquisas e Apoio aos Métodos de Survey.

Todas estas referências e o uso da matemática nas ciências sociais, não obstante, são também projetadas a críticas de diversas fontes. A perspectiva hermenêutica pode ser aqui um exemplo razoável de como teóricos tentaram organizar e limitar o papel da matemática nas ciências sociais. A Escola de Frank- furt e os escritos de Adorno e Horkheimer seriam bons exemplos de reflexões sobre a aplicação de métodos tão íntimos às ciências naturais. Heidegger, em "Basic Writtings" (1977) mostra como não apenas os números são importantes à investigação social, bem como o caminho de aprendizagem crítica a esses.

Raymond Boudon, em "Métodos da sociologia" (1971), especifica-nos a partir das reflexões sobre métodos quantitativos e qualitativos que as ciências sociais e humanas ocupam-se de problemas cujas características lógicas são muito diferentes, implicando em métodos muito diversos (por isso fala em Métodos, no plural). Eis um ponto de reflexão que baliza as diversas contribuições metodológicas que encenam de acordo com os problemas diversos aos quais se dedicam os cientistas sociais, no que a compreensão não seria a análise em si, mas apenas uma das fases de pesquisa.

Dentro desse quadro histórico mais geral, pensemos sobre casos mais específicos dos moldes de investigação contemporâneos: as contribuições dos paradigmas qualitativo e quantitativo.

\section{MÉTODOS QUANTITATIVOS E A CORRIDA PELOS NÚMEROS}

A começar pelo avesso, coloquemos alguns dos preconceitos mais corriqueiros na aplicação do paradigma quantitativo nas ciências sociais e humanas. Tais métodos são usualmente rotulados de positivistas, mecanicistas, áridos, inflexíveis e deterministas, a sustentar uma desconfiança da informação numérica. Porém, por traz destes aspectos de "rejeição", o que temos, em verdade, é o método quantitativo como estabelecedor de comportamentos que podem ser medidos através de números. Seu instrumento preferencial é o questionário na quantificação de fenômenos para aná-

lises posteriores. É comum observarmos a metodologia quantitativa em arcabouço mais sofisticado em outras áreas de conhecimento, todavia, a utilização cada vez mais usual de rígidos meios computacionais tem facilitado seu emprego nas ciências humanas e sociais. Programas computacionais como o SPSS, PSPP, Minitab, Excell, HLM, dentre muitos outros, realizam cálculos precisos e já auxiliam na visualização de potencialidades de bancos de dados. 0 que há algumas décadas colocava-se como tarefa árdua ao pesquisador, o cálculo de coeficientes de correlação, significâncias estatísti- 
cas, dentre outros resultados das estatísticas descritiva e inferencial, hoje mostra-se como ferramenta mais acessível às mãos do pesquisador social, ocupado em estabelecer sentido para as inferências desenvolvidas.

Os investigadores quantitativos tendem a utilizar um conjunto de análises estatísticas e generalizações para determinar o padrão dos dados e o seu significado. Diferentemente do que versa parte dos preconceitos mencionados, possuem flexibilidade no tratamento das informações ao escolherem, via um projeto de pesquisa anterior, uma linha metodológica de observação para informações complexas. Em determinadas culturas acadêmicas, as metodologias quantitativas são mais utilizadas do que em outras. Essa variação pode ser originada pelos departamentos em que se inserem tais pesquisas, pelas universidades, pelos locais de investigação etc. Por exemplo, em um ambiente acadêmico com reduzida produção de pesquisas quantitativas é esperado que a formação docente na orientação de projetos do gênero seja igualmente reduzida. Entretanto, ponto articuladamente determinante nestes processos é o tipo de objeto de observação escolhido para análise. A escolha do método não relaciona-se apenas às preferências e possibilidades de alcance do investigador, mas sim, à articulação

\section{MÉTODOS QUALITATIVOS E OS SENTIDOS DAS PALAVRAS}

Noutra perspectiva, temos as metodologias qualitativas, por vezes taxadas de imprecisas, não científicas ou não objetivas. Os limites das pretensões destas páginas não resultarão em discussões sobre objetividade das ciências sociais, mas cabe considerarmos que não limitamos objetividade metodológica a aspectos de propriedades meramente numéricas.

A pesquisa qualitativa estabelece padrões de comportamentos verificados através de fatos observáveis. concatenada entre as partes componentes de um projeto que antecipa as escolhas metodológicas para as respostas das perguntas de investigação.

Notadamente, o paradigma quantitativo por muitas vezes torna-se um caminho árduo quando, por exemplo, não conseguimos isolar uma determinada variável da realidade para nossas matrizes de dados, ou quando não conseguimos uma homogeneização necessária para realização de testes de máxima verossimilhança, bem como detectamos variáveis "parasitas" ou "invisíveis" que, em vez de recusarem ou aceitarem as hipóteses experimentais de trabalho, findam por reelaborarem os processos de investigação. Estes episódios são comuns não apenas de acordo com os tipos de pesquisa realizados, mas também, giram consoante às experiências empíricas do investigador envolvido na elaboração de meios de aferição da realidade. Por vezes, encontramos dados em diferentes magnitudes e assimilações terminológicas, resultados de unidades de medida díspares mas, quando em situações específicas, resultados de diferenças culturais próprias às temáticas da ciências sociais e humanas. Eis quando a experiência empírica, associada ao arcabouço teórico do cientista social, faz-se determinante no processo de conhecimento e análise social.
Sua ferramenta preferencial é a entrevista e pode ter como instrumento de recolha os estudos de caso, as observações participante e direta, a formação de grupos de focais em análises temáticas, as histórias de vida, as histórias orais, as biografias etc. Para o tratamento das informações, as análises de discurso, de conteúdo ou de conjuntura são as mais tencionadas no meio acadêmico das humanidades. Na perspectiva qualitativa, nota-se uma unidade indissociável entre o metodológico e o epistemológico, isto é, entre a 
produção e a elaboração do conhecimento. É ela mais sensível na análise de fenômenos ocultos, bem como na inclusão da subjetividade e do reconhecimento de processos de alteridade na realidade social. Destaca e releva elementos característicos da natureza humana que metodologias quantificadoras por vezes têm dificuldade de acessar (Boudon, 1971).

0 recurso qualitativo apresenta-se como adequado notadamente ao estudo de fenômenos particulares ou quando se tem por objetivo as origens e as razões de tais fenômenos. Não podemos precisar quando o estudo qualitativo torna-se mais viável financeiramente para execução, todavia, ele tende a ser mais acessível do que o quantitativo na maioria das vezes, posto sua característica de análises particulares, centradas, e que necessitam de equipe reduzida nas fases de recolha de informações. Estudos de caso, por exemplo, permitem uma finura de observação maior, bem como a intensidade da observação pode suprir uma dada amplitude de estudo de uma suposta investigação quantitativa.

\section{PARA ENCERRAR COMEÇANDO: OPÇÕES METO- DOLÓGICAS NO COTIDIANO DA INVESTIGAÇÃO}

A escolha por um dos paradigmas no momento de elaboração do projeto de pesquisa muitas vezes finda por ocasionar disputas epistemológicas acirradas e desnecessárias, como se tais paradigmas fossem incompatíveis. A metodologia mista, por exemplo, vem propor a coexistência destes modos de fazer no processo de investigação, ou seja, propõe parcerias. Os processos qualitativo e quantitativo não se excluem mutuamente mas são, antes, categorias mutuamente inclusivas desde que os propósitos de utilização estejam afinados aos objetivos de trabalho. Eis quando quantidade se transforma em qualidade e, qualidade, se transforma em quantidade. Em outros termos, e para refrisarmos as interconexões, temos que a natureza de um objeto não se opõe à quantidade. A noção de quantidade sempre pressupõe a noção de qualidade e toda qualidade é quantificável. Medir algo significa tornar possível a repetição de uma qualidade (Boudon, 1971).

A multi e a interdisciplinaridade funcionam como aportes ao acesso mais amplo da coleta de informações. Um estudo de natureza originalmente qualitativa, em que fosse aplicado o recurso da análise de discurso no tratamento das informações poderia, em uma segunda instância, ampliar por um estudo quantitativo uma análise de regressão a responder quais variáveis de um conjunto $X$ de dimensões analíticas explicam a variação da variável dependente de análise. Os achados de pesquisa podem complementar conclusões e respostas aos problemas tratados, refutar ou aprofundar noções mais sensíveis de visualização por uma ou outra técnica de investigação. Noutro exemplo, poderíamos ter um estudo quantitativo em que seus resultados isolariam determinadas variáveis independentes como resposta à variação de uma observada variável dependente. Um estudo qualitativo posterior poderia aprofundar as razões daqueles achados, bem como dar sentido ao modelo resultante da análise quantitativa. E isso, para não falarmos das contribuições de estudos de origem exploratória que, tanto em uma como em outra perspectiva, podem ceder campo para estudos mais pontuais quando, e aí mais um exemplo, a aplicação de questionários numa amostragem não representativa já indica aspectos da realidade que venham ser tratados pontualmente em estágio ulterior numa história oral, notadamente qualitativa. Por que haveríamos de engessar a possibilidade de a partir de uma análise de clusters (quantitativa) chegarmos a uma análise de conteúdo (qualitativa)? 
O uso conjunto destas metodologias pode, então, ter um tom exploratório, ou de levantamento de dados secundários (e aí a necessária multidisciplinaridade), conquanto sejam percebidas as contribuições de cada ferramenta metodológica nas singularidades das problemáticas tratadas.

A partir da possibilidade de mesclas e da liberdade acadêmica/criativa de que fazemos uso, percebemos que os paradigmas qualitativo e quantitativo dependem diretamente do problema de pesquisa. Quais condições permitem ao pesquisador ter acesso à realidade social? Método significa Linguagem. Há potencialidades e limitações nas duas abordagens. A pesquisa qualitativa ocorre de maneira menos unificada do que a quantitativa, embora nas ciências sociais não exista um método único de trabalho, mas sim, métodos, no plural. A suposta tentativa de unificação de outra então imaginada metodologia das ciências sociais estaria fadada ao fracasso da padro- nização do que é plural, plástico e relativo. A eficácia das ciências sociais não está na unidade haja vista a diversidade de objetos que considera. De igual modo, nenhum dos paradigmas aqui tratados é análise em si. São, e apenas, instrumentos que preparam o terreno para análise porque recortam as etapas de métodos e técnicas de pesquisa.

Por fim, frisamos a importância das condições de domínio por parte dos investigadores em ambas metodologias. Ainda que o pesquisador apoie-se mais em uma ou em outra perspectiva, ter as propriedades mínimas adequadas à leitura e compreensão de pesquisas realizadas por outrem é parte integrativa da formação do cientista social. Nenhum dos paradigmas é mais científico que outro, todavia, para exercício da ciência, a manipulação consciente e responsável de realização, leitura e análise, fazem-se necessárias para o estoque de conhecimento da profissão de investigador e/ou docente.

\section{REFERÊNCIAS}

BOUDON, Raymond. Métodos da Sociologia. 2.ed., Rio de Janeiro: Vozes, 1971.

CHALMERS, Alan. A Fabricação da Ciência. São Paulo: UNESP, 1994.

FERREIRA, Jonatas. “A Produção de memória biotecnológica e suas consequências culturais”. In: Revista Brasileira de Ciências Sociais, Vol. 18, No: 53, 2003.

HEIDEGGER, Martin. Basic Writtings. New York, 1977.

WILSON, Thomas. “Sociologia e Método Matemático”. In: GIDDENS, Anthony; TURNER, Jonathan (orgs). Teoria Social Hoje. São Paulo: UNESP, 1999. 
1 Cientista Político. Prof. Dr. do Núcleo de Pós-Graduação em Ciências Sociais da Faculdade Integrada Tiradentes (NPGCS-FITs), Maceió-AL, Brasil. Contato: diego_freitas@fits.edu.br

2 Socióloga. Profa. Dra. do Núcleo de Pós-Graduação em Ciências Sociais da Faculdade Integrada Tiradentes (NPGCS-FITs), Maceió-AL, Brasil. Contato: marina_felix@fits.edu.br

Aceito em: 13 de julho de 2013

3 Cientista Política. Profa. Dra. do Núcleo de Pós-Graduação em Ciências Sociais da Faculdade Integrada Tiradentes (NPGCS-FITs), Maceió-AL, Brasil. Contato: lorena_monteiro@fits.edu.br 